\title{
Journal of Intelligent Industrial Systems: Preface to Volume 1 Issue No 4
}

\author{
Gerasimos Rigatos $^{1} \cdot$ Pierluigi Siano $^{2}$
}

Published online: 20 November 2015

(C) Springer Science+Business Media Singapore 2015

The fourth issue of the first volume of the Journal of Intelligent Industrial Systems provides an insight to methods and techniques that improve intelligence, autonomy and reliability of benchmark industrial systems. Actually, two main classes of research results are considered. The first one is control theory-oriented while the second one is focused on electric power systems technology. In particular, in the area of control systems the issue presents (i) a new nonlinear control and filtering methods for unmanned aerial vehicles, (ii) an overview of approaches for the autonomous navigation and localization of robotic vehicles, and (iii) an evolutionary optimization method for solving the problem of reduced order modelling of multi-variable linear dynamical systems. In the area of electric power systems research the issue presents new findings on (i) global linearization-based control and filtering for high voltage DC power transmission systems, (ii) knowledge-based methods for home energy management systems and (iii) probabilistic modelling for improving the integration of distributed photovoltaic units in the lowvoltage electricity grid. Finally, in complement to control and electric power systems topics, an article is published on the use of visual inspection techniques for automated defect classification in production lines.

In article Control of quadrotors with the use of the Derivative-free nonlinear Kalman Filter by G. Rigatos and P. Siano, the Derivative-free nonlinear Kalman Filter is used

Pierluigi Siano

psiano@unisa.it

Gerasimos Rigatos

grigat@ieee.org

1 Unit of Industrial Automation, Industrial Systems Institute, 26504 Rion Patras, Greece

2 Department of Industrial Engineering, University of Salerno, Fisciano 84084, Italy for developing a robust controller which can be applied to quadropters. The control problem for quadropters is nontrivial and becomes further complicated if this robot is subjected to model uncertainties and external disturbances. Using differential flatness theory it is shown that the model of a quadropter can be transformed to linear canonical form. For the linearized equivalent of the quadropter it is shown that a state feedback controller can be designed. Since certain elements of the state vector of the linearized system can not be measured directly, it is proposed to estimate them with the use of a novel filtering method, the so-called Derivativefree nonlinear Kalman Filter. Moreover, by redesigning the Kalman Filter as a disturbance observer, it is is shown that one can estimate simultaneously external disturbances terms that affect the quadropter or disturbance terms which are associated with parametric uncertainty.

In article An overview on visual odometry and visual SLAM by K.Yousif et al., an overview is provided about key problems and about the associated solutions in mobile robot localization and mapping. Main issues in autonomous robot navigation are discussed and a review is given about of the state-of-the-art techniques for mobile robots localization and mapping. The article covers both common localization techniques such as wheel odometry and dead reckoning, as well as advanced methods such as Visual Odometry (VO) and Simultaneous Localization and Mapping (SLAM) techniques. $\mathrm{VO}$ is analyzed for both monocular and stereo vision systems using feature matching/tracking and optical flow techniques. The authors discuss and compare the basics of most common SLAM methods based on nonlinear Kalman and Particle Filtering approaches. They also focus on implementation issues of visual navigation methods such as feature extraction, feature matching, outlier removal and data association techniques. 
In article Application and comparative analysis of various classical and soft computing techniques for model reduction of MIMO systems, by U. Salma and K. Vaisakh, the need for simplifying the models of multivariable dynamical system's is shown. The objective is to arrive at transfer function descriptions consisting of low order numerator and denominator polynomials. Some existing classical model reduction techniques for multivariable system are considered and compared for their performance. The truncated model's accuracy is measured with criteria such as the overall minimum integral square error (ISE), the integral absolute error (IAE) and the integral time absolute error (ITAE). Actually, a model reduction approach based on multi-objective optimization is developed. The aim is to find a Pareto-optimal solution for criteria like ISE, IAE and ITAE and to this end particle swarm optimization and differential evolution (MOPSO \& MODE) methods are used. The obtained reduced order models are shown to better approximate the exact models of the studied dynamical systems. As a case study, reduction of a 10th order multivariable linear time invariant power system model is considered. It is also demonstrated that evolutionary algorithms have improved performance in this optimization problem comparing to particle swarm methods.

In article $A$ global linearization approach to control and state estimation of a voltage source converter-HVDC system by G. Rigatos and P. Siano, a control method is developed for the VSC-HVDC system, that is for an AC to DC voltage source converter connected to the electricity grid through a high voltage DC transmission line terminating at an inverter. By showing that the VSC-HVDC system is a differentially flat one, its transformation to the linear canonical form becomes possible. This is a global input-output linearization procedure that results into an equivalent dynamic model of the VSC-HVDC system for which the design of a state feedback controller is enabled. Moreover, to estimate and compensate for modeling uncertainty terms and perturbation inputs exerted on the VSC-HVDC model it is proposed to include in the control loop a disturbance observer that is based on the Derivative-free nonlinear Kalman Filter. The performance of the proposed VSC-HVDC control scheme is evaluated through simulation experiments.

In article Impacts of house sizes, appliance ratings and usage patterns on demand response applications by $\mathrm{M}$. Kuzlu et al. it is shown how a Home Energy Management (HEM) system can facilitate automated demand response (DR) participation for residential customers. The aim of the article is to analyze the impact of different house sizes, appliance ratings and usage patterns on residential DR implementation. To this end the authors exploit empirical knowledge formulated as a set of predefined DR strategies. In particular, several simulation studies are conducted to demonstrate differences in the HEM system performance, when implemented in households that have different characteristics.
Results show that houses of different sizes, appliance ratings and usage patterns have different impacts on residents' comfort, and determine the feasible levels of DR implementation.

In article Monte Carlo-based stochastic analysis results for coordination of single-phase rooftop PVs in low voltage residential networks by $\mathrm{N}$. Safitri et al., it is shown that power grid performance and electric power quality may deteriorate due to the unplanned installation of single-phase rooftop photovoltaic (PV) systems in low voltage (LV) residential feeders. Actually, the empirical selection of location and ratings for such DC power generation units may cause reversed power flows, high losses and unacceptable voltage profiles in the electricity grid. As a first measure against this power quality drop limitations have been imposed about the maximum allowable number of PVs in LV networks. To overcome these issues in the integration of photovoltaic units in the power grid, the article investigates the performance of a communication-based and intelligent voltage profile regulating technique under a Monte Carlo-based stochastic framework. This technique is applicable to $\mathrm{LV}$ residential feeders with single-phase rooftop PVs and relies on the availability of smart meters along the LV feeder. so as to transmit phase voltage measurements to the controllers of the PV inverters. The objective of the voltage regulation technique is to minimize voltage unbalance along the feeder. The effectiveness of the voltage regulation technique are investigated in this paper by the help of MATLAB-based simulation studies.

Finally, in article Automatic defect classification on a production line by N. Borghese and M. Fomasi a novel defect classification system is defined that works in real-time on the images of material running on the production line provided by a video-inspection module. The classifier consists of a two-stage hierarchical architecture: in the first stage an adequate number of features is extracted from the image, while in the second stage labeling of the defect image is carried out from these features. Features design is also a two-stage process: in the first stage, standard features are defined with the help of the domain expert, while in the second stage more refined features are introduced to improve the classification. These are based on a local voting mechanism aiming at characterizing threads, and on matched filters and the co-occurrence matrix to identify defects. Statistical analysis allows a pruning of the number of features, leaving only the most significant ones. An analysis of the classifier's output distribution is used to guarantee that all dangerous defects are always detected.

The topics treated in the aforementioned articles come to confirm that the area of intelligent industrial systems is a multi-disciplinary one and combines both analytical methods and computational tools for improving the performance of robotic systems, industrial production, and energy man- 
agement. It has shown that control theoretic methods used in robotic and industrial systems become much more effective if combined with intelligent computation techniques which enable to compensate for modelling uncertainties and missing sensory information. Moreover, it has shown that the use of intelligence-based optimization and management tools in electric power systems can result into the improvement of the quality of the produced electric power and into a more profitable and reliable functioning of the electricity grid. As there is a constant growth in the need for industrial systems' operation under changing conditions and model variations, one should also anticipate further development of the aforementioned control and modelling techniques.

Dr. G. Rigatos and Dr. P. Siano

Editors-in-Chief 